\title{
Dissipative Particle Dynamics Simulations of Polymersomes
}

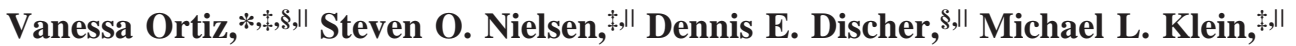 \\ Reinhard Lipowsky, ${ }^{\dagger}$ and Julian Shillcock ${ }^{\dagger}$ \\ Max Planck Institute of Colloids and Interfaces Golm, D-14424 Potsdam, Germany, Center for Molecular \\ Modeling, Department of Chemistry, Biophysical Engineering Lab, Department of Chemical \& Biomolecular \\ Engineering, and Laboratory for Research on the Structure of Matter, University of Pennsylvania, \\ Philadelphia, Pennsylvania 19104-6202
}

Received: March 11, 2005; In Final Form: July 25, 2005

\begin{abstract}
A DPD model of PEO-based block copolymer vesicles in water is developed by introducing a new density based coarse graining and by using experimental data for interfacial tension. Simulated as a membrane patch, the DPD model is in excellent agreement with experimental data for both the area expansion modulus and the scaling of hydrophobic core thickness with molecular weight. Rupture simulations of polymer vesicles, or "polymersomes", are presented to illustrate the system sizes feasible with DPD. The results should provide guidance for theoretical derivations of scaling laws and also illustrate how spherical polymer vesicles might be studied in simulation.
\end{abstract}

\section{Introduction}

Membranes composed of pure phospholipid have been studied since the $1960 \mathrm{~s}^{1,2}$ and simulated in atomistic detail for about a decade. ${ }^{3}$ Membranes composed of purely synthetic polymer have been studied experimentally for less than a decade ${ }^{4-10}$ and, because of the higher molecular weight $(M)$ of the amphiphilic polymers $(>\mathrm{kDa})$ compared to lipids $(<\mathrm{kDa})$, polymer membranes have only recently been simulated. ${ }^{11-13}$ In practice, polymer vesicles appear to have advantages over lipid vesicles because the physicochemical properties of "polymersomes" are more widely tunable. Specifically, the membrane elasticity, stability, permeability, and thickness are easily controlled by varying the molecular weight of the blocks. In addition, controlled degradability can be incorporated by the addition of hydrolyzable monomers. Sufficiently hydrophilic monomers can form blocks with variable hydrophobicity based on the molecular weight, namely at low $M$ the block will be water soluble but at high $M$ it can form the hydrophobic core of diblock (selfassembling) vesicles. ${ }^{14}$

As with lipids, the more hydrophobic segments of amphiphilic block copolymers exclude water and form a melt whereas the hydrophilic chains hydrate. This aqueous environment alters the behavior from diblock melts, which historically have been the focus of theoretical efforts. Although theoretical discussions of polymer membranes and property scaling ${ }^{15}$ predate even the first experiments, ${ }^{10}$ these have always been based on the melt derivations. Indeed, now that the variability in structure and properties of polymer membranes with $M$ is beginning to be elucidated experimentally, ${ }^{16-21}$ it has become apparent that the theory does not adequately describe aqueous systems. The essential physical properties of these systems are due to the

* Address correspondence to this author. E-mail: vaortiz@seas.upenn.edu.

Max Planck Institute of Colloids and Interfaces Golm.

$\doteqdot$ Center for Molecular Modeling, Department of Chemistry, University of Pennsylvania.

$\S$ Biophysical Engineering Lab, Department of Chemical \& Biomolecular Engineering, University of Pennsylvania.

"Laboratory for Research on the Structure of Matter, University of Pennsylvania. balance between the tendency of the components to phase separate into a hydrated component and a melt, and the chemical bond attaching the blocks together. A simple model that only incorporates these effects and is still capable of capturing the experimental scaling relations would be of great value in developing a theoretical description.

Recent simulations of polymer membranes have exploited coarse grained molecular dynamics (CGMD) ${ }^{12,13}$ but have proven unable to span much of the $M$ range of experiments let alone demonstrate vesicle formation. Simulations of systems whose natural length scales far exceed those of traditional atomistic computations is perhaps better met today by the method of dissipative particle dynamics (DPD). ${ }^{22}$ The interaction parameters for DPD simulations consist of one parameter for each species that determines the single component fluid's compressibility, an intramolecular part capturing the connectivity, and an intermolecular part that uses a single parameter for each pair of species to describe their mutual solubility. The link between the tendency of the species to phase separate and their mutual solubility is provided by the liquid-liquid interfacial tension that can be used directly as a fitting parameter. ${ }^{23}$ While a CGMD model with a mapping of approximately three heavy atoms for every coarse grained site offers a speedup of $10^{5}$ over fully atomistic molecular dynamics (AAMD), ${ }^{24}$ the same mapping in the DPD framework provides an increase in efficiency of $10^{6}$ largely because the interactions have a shorter cutoff. Figure 1 illustrates the length scales accessible to the different techniques with the above mapping at reasonable computational cost for a moderate molecular weight diblock copolymer. In DPD, this copolymer can make a closed vesicle.

Here we have constructed a model in the DPD framework for aqueous poly(ethylene oxide)-polyethylethylene (PEO$\mathrm{PEE}$ ) diblock copolymer vesicles by incorporating intramolecular data from atomistic simulations and intermolecular data from experimental interfacial tensions. Elastic properties of the membrane were used to evaluate two coarse graining schemes for DPD. The conventional coarse graining of three water molecules and one monomer per interaction site gave unphysical results, but elasticities obtained with a new mapping based on 


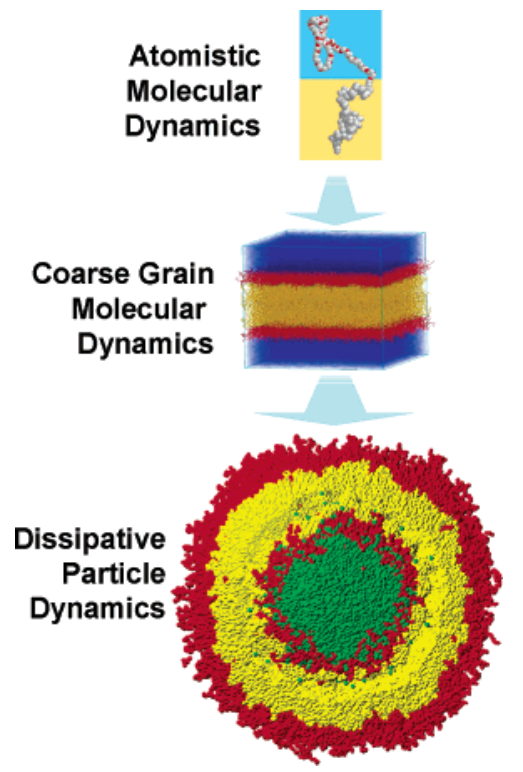

Figure 1. Length scales and structures accessible to AAMD, CGMD, and DPD techniques for high molecular weight diblock copolymers. CGMD offers an increase in efficiency over AAMD of $\sim 10^{5}$, while DPD gives an increase of $\sim 10^{6}$.

pure liquid densities agreed with experimental data. In particular, using this new mapping, the scaling of the hydrophobic core thickness of the membrane with $M$ was found to be in agreement with experiment. Finally, preliminary studies on stretched vesicles are shown in order to explore the time and length scales of rupture.

\section{Methods}

DPD simulation methods are increasingly being applied to a wide range of problems, from lipid membranes and vesicles ${ }^{25-27}$ to polymer-polymer surface tension calculations, ${ }^{28}$ to diblock copolymer melt microphase separation, ${ }^{29}$ and to studies of shear flow on solid surfaces. ${ }^{30}$ In simulating polymer-based membranes as well as vesicles in water here, we exploit the system size advantages of DPD. The canonical algorithm ${ }^{31-35}$ has been used with the exception of a modification on the atomistic-tocoarse-grained mapping, which is presented in section III. Briefly, soft beads representing a volume of fluid containing several molecules or molecular fragments interact via effective forces chosen so as to reproduce the compressibility of water and mutual solubility of the different species. The functional forms of the effective forces are as described in the DPD literature, ${ }^{31-35}$

conservative force, $F_{i j}^{\mathrm{C}}= \begin{cases}a_{i j}\left(1-r_{i j} / r_{0}\right) \hat{r}_{i j} & \text { for } r<r_{0} \\ 0 & \text { for } r \geq r_{0}\end{cases}$ dissipative force, $F_{i j}^{\mathrm{D}}=$

$$
\begin{cases}-\gamma\left(1-r_{i j} / r_{0}\right)^{2}\left(\hat{r}_{i j} \cdot v_{i j}\right) \hat{r}_{i j} & \text { for } r<r_{0} \\ 0 & \text { for } r \geq r_{0}\end{cases}
$$

random force, $F_{i j}^{\mathrm{R}}= \begin{cases}\sigma\left(1-r_{i j} / r_{0}\right) \xi_{i j} \hat{r}_{i j} & \text { for } r<r_{0} \\ 0 & \text { for } r \geq r_{0}\end{cases}$

The mass $\left(m_{0}\right)$ and length $\left(r_{0}\right)$ scales, as well as the selfrepulsion parameters $\left(a_{i i}\right)$ in the simulations are set by the mapping chosen for the water of 3 molecules per bead. The
TABLE 1: Systems Simulated in the DPD Framework Consisting of Hydrated Bilayers of Copolymers of Different Length but Constant Hydrophilic Fraction ${ }^{a}$

\begin{tabular}{|c|c|c|c|c|}
\hline diblock & box size $(\mathrm{A})$ & $\begin{array}{c}\text { area per } \\
\text { molecule } \\
\left(\AA^{2}\right)\end{array}$ & $\begin{array}{l}\text { no. of } \\
\text { chains }\end{array}$ & $\begin{array}{c}\text { no. of } \\
\text { water } \\
\text { molecules }\end{array}$ \\
\hline \multirow[t]{7}{*}{ PEO40-PEE37 } & $207 \times 207 \times 259$ & 104.26 & 822 & 59585 \\
\hline & & 113.06 & 758 & 64513 \\
\hline & & 121.39 & 706 & 68517 \\
\hline & & 125.66 & 682 & 70365 \\
\hline & & 129.85 & 660 & 72059 \\
\hline & & 138.22 & 620 & 75139 \\
\hline & & 146.49 & 585 & 77834 \\
\hline \multirow[t]{2}{*}{ PEO60-PEE55 } & $271 \times 271 \times 323$ & 157.09 & 935 & 157074 \\
\hline & & 161.05 & 912 & 159719 \\
\hline \multirow[t]{2}{*}{ PEO80-PEE74 } & $330 \times 330 \times 381$ & 177.94 & 1224 & 273104 \\
\hline & & 182.56 & 1193 & 277695 \\
\hline \multirow[t]{2}{*}{ PEO100-PEE93 } & $381 \times 381 \times 433$ & 198.85 & 1460 & 419360 \\
\hline & & 203.16 & 1429 & 425121 \\
\hline \multirow[t]{2}{*}{ PEO120-PEE111 } & $427 \times 427 \times 478$ & 219.01 & 1665 & 585518 \\
\hline & & 223.30 & 1633 & 593074 \\
\hline
\end{tabular}

${ }^{a}$ The chain lengths were chosen to span the range of molecular weight sampled in experiments. Systems at different area per molecule are needed to extract the zero tension state in the NVT ensemble.

dissipation strength, $\gamma$, is set as 4.5 (in units of $\sqrt{m_{0} k_{\mathrm{B}} T / r_{0}{ }^{2}}$ ) and a value of 3 is used for the random noise strength, $\sigma$, to control the temperature, $k_{\mathrm{B}} T$, to a unit value via the fluctuation dissipation theorem $\left(\sigma^{2}=2 \gamma k_{\mathrm{B}} T\right)$. The relative particle velocity, $v_{i j}$, is defined as $\left(v_{i}-v_{j}\right)$ and $\xi_{i j}$ is a random variable with zero mean and unit variance. A constant time step of 0.02 (in units of $\sqrt{m_{0} r_{0}{ }^{2} / k_{\mathrm{B}} T}$ ) was used for both the model parametrization and the molecular weight studies, maintaining in this way consistency between the system information incorporated into the model and the information extracted from it. Furthermore, it was recently shown by Jakobsen and co-workers, ${ }^{36}$ by using softer potentials than the ones used in this work, that time steps varying by 5 -fold around 0.02 introduce less than $20 \%$ deviations in membrane tension. These do not seem significant enough to alter our conclusions here. The standard value ${ }^{28}$ of 0.5 is used for the empirical factor $\lambda$ in the DPD velocity Verlet algorithm. The DPD simulations were performed on hydrated planar bilayers made of PEO-PEE diblock copolymers of different lengths (denoted PEOm-PEE $n$ where $m$ and $n$ are the number of monomers) but a constant hydrophilic ratio of 0.46 . The chain lengths were chosen to span the entire range of molecular weight sampled in experiments. The systems are reported in Table 1. The simulations were each run for 200000 time steps.

In this study, the DPD simulations are performed in the NVT ensemble; therefore a series of runs must be performed for each system at different area per molecule in order to find the zero tension state. First, the smallest system of PEO40-PEE37 was run for 7 different area per molecule (see Table 1) from which the surface tension was extracted, according to Goetz and Lipowsky, ${ }^{37}$ by integrating the stress tensor along the dimension normal to the bilayer. A plot of tension versus area-per-molecule was constructed for this system, from which the zero tension area was extracted by linear interpolation. To obtain the zero tension state for the other diblock copolymer lengths an initial prediction was made by assuming a scaling of hydrophobic core thickness, $d$, with molecular weight, $M$, of $d \sim M^{0.5}$ as suggested by Bermudez et al. ${ }^{16}$ (by assuming incompressibility, the area should also scale with an exponent of 0.5 ). Two simulations were then performed for each chain length at values of area per molecule around the predicted value, and the DPD-derived 


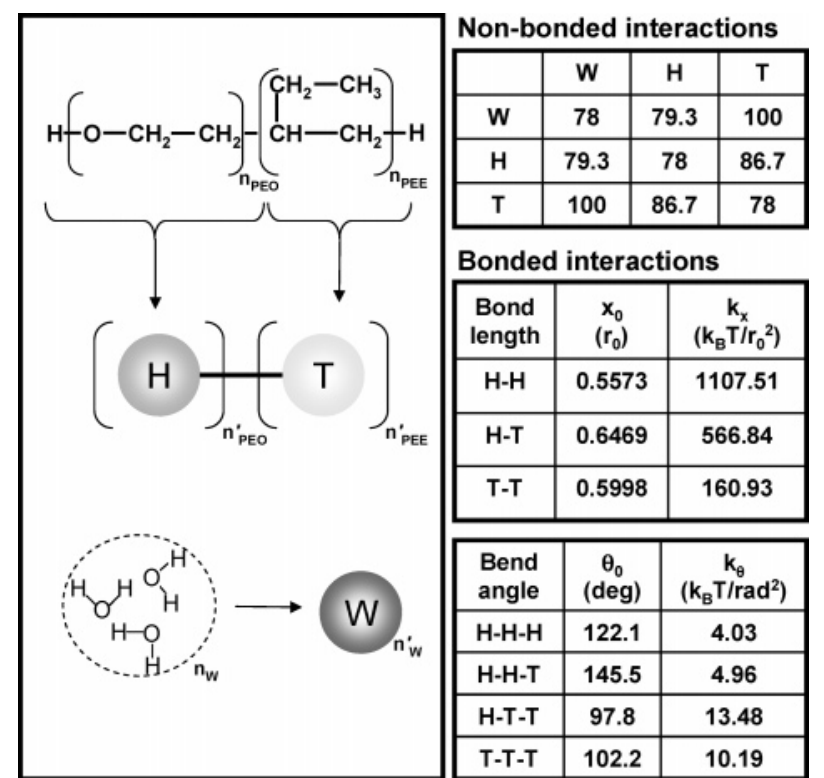

Figure 2. Schematic drawing of the mapping from atomistic to DPD. The number of PEO and PEE groups are denoted by $n_{\mathrm{PEO}}$ and $n_{\mathrm{PEE}}$, respectively, and the number of the corresponding $\mathrm{H}$ - and T-beads by

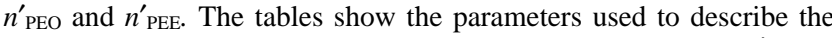
interactions between the DPD sites, which are given for the $n_{\text {PEO }}^{\prime}=$ $n_{\mathrm{PEO}} / 1.392, n_{\mathrm{PEE}}^{\prime}=n_{\mathrm{PEE}} / 0.774$ mapping of eq 1 . This change results in a DPD model leading to more physical properties comparable to experimental data versus 1:1 mappings.

$d$ was obtain by substituting the zero tension area into a fit of the thickness versus area data.

For the vesicle rupture simulations a cubic box of $(51.7 \mathrm{~nm})^{3}$ was used, which for a particle density of three consists of $\sim 1.5$ million sites. The vesicles were constructed by evenly distributing the total number of PEO40-PEE37 diblocks (1569) over the two leaflets such that the area per molecule is fixed at 3.75 $\mathrm{nm}^{2}$ (about three times the zero tension value), giving an inner leaflet with 314 chains and a radius of $\sim 10 \mathrm{~nm}$, and an outer leaflet with 1255 chains and a radius of $\sim 20 \mathrm{~nm}$. This size is at the lower end of the experimental range for polymersomes.

\section{Model Construction}

Two mappings are presented in section IIIa. The optimization of the DPD model parameters for these two mappings involves the intra- and intermolecular parameters. In section IIIb the intramolecular parameters are determined with a reasonable guess for the intermolecular parameters. After the intramolecular parameters have been optimized, the intermolecular parameters are determined in section IIIc. A final check was done to verify that the intramolecular parameters did not change upon refinement of the intermolecular parameters, which is to be expected because the intramolecular interactions dominate the potential energy.

III.a. Coarse Grained Mapping. It is conventional in the DPD framework to base the coarse grain mapping on that of water. ${ }^{38}$ Namely, the amount of matter contained in each DPD site is constant for all species and is taken as that of the mapping chosen for the water. This means that, with the standard mapping of three water molecules per DPD site, ${ }^{38}$ the coarse graining for PEO and PEE gives the conventional mapping of approximately one monomer per site: in Figure 2 this would mean that $n_{\mathrm{PEO}}^{\prime}=n_{\mathrm{PEO}}$ and $n_{\mathrm{PEE}}^{\prime}=n_{\mathrm{PEE}}$. However, this approach was found to be unsatisfactory for two reasons. First, the hydrophobic core density profile is much too narrow because the hydrocarbon block has the same bulk density as water. Second, attempts to obtain tension versus area per molecule curves, as described in the Methods section, resulted in unphysical values for the membrane elasticity (data not shown).

The root cause of these problems is in the exclusive use of water to define all aspects of the DPD description, from liquid compressibilities to diffusion time scales, to the density of the beads. To make a stronger link with experiments, the constraint of constant density of the beads for all species was removed. The resulting coarse graining hence varies with species identity. This variation is chosen such that the bulk density of the pure species matches experimental data. The new density mapping obtained with this approach is given in Figure 2 by

$$
\begin{aligned}
n_{\mathrm{PEO}}^{\prime} & \equiv n_{\mathrm{PEO}} / m_{\mathrm{PEO}} \\
n_{\mathrm{PEE}}^{\prime} & \equiv n_{\mathrm{PEE}} / m_{\mathrm{PEE}} \\
n_{\mathrm{W}}^{\prime} & \equiv n_{\mathrm{W}} / m_{\mathrm{W}}
\end{aligned}
$$

where the mapping factors were found to be

$$
m= \begin{cases}m_{\mathrm{PEO}}=1.392 & \text { monomers/bead for PEO } \\ m_{\mathrm{PEE}}=0.774 & \text { monomers/bead for PEE } \\ m_{\mathrm{W}}=3.01 & \text { molecules/bead for water }\end{cases}
$$

III.b. Intramolecular Interactions. The intramolecular parameters for the two mappings cannot be separately determined because the density mapping corresponds to an unnatural division of the underlying atomistic structures. In this section, the development of the parameters is given for the conventional mapping, and then the underlying data are converted to the density mapping.

The intramolecular interactions in the DPD scheme are described by harmonic bond lengths and bend angles. The equilibrium values $\left(x_{0}\right.$ and $\left.\theta_{0}\right)$ and force constants $\left(k_{x}\right.$ and $\left.k_{\theta}\right)$ are chosen to reproduce the mean $\left(l^{\prime}\right.$ and $\left.\theta^{\prime}\right)$ and standard deviation of the target bond length and bend angle distributions, which were obtained from AAMD simulations by defining the position of the coarse grain site as the center of mass of the group of atoms included in that site. To clarify the relationship between $l^{\prime}$ and $x_{0}$ (or $\theta^{\prime}$ and $\theta_{0}$ ) it is noted that $l^{\prime}$ is the first moment of the target distribution while $x_{0}$ is the value of the equilibrium length given as an input parameter for the DPD simulation. The mean value of the distribution of bond lengths from the simulation is collected and the value of $x_{0}$ is adjusted accordingly to bring the mean in agreement with the target value $l^{\prime}$. For the density mapping the positions of the coarse grain sites cannot be determined accurately because the coarse graining involves accounting for fractions of monomers and sometimes fractions of atoms. In what follows it is shown how the density mapping target bond length and bend angle distributions are obtained from those of the conventional mapping.

Given the bond length $l$ and the preferred bend angle $\theta$, for the conventional mapping, we need to determine the bond length $l^{\prime}$ and the preferred bend angle $\theta^{\prime}$ for the density mapping without changing the physical properties of the polymer. In the absence of intermolecular interactions the physical properties are characterized by the end-to-end distance distribution. We appeal to the two simplest polymer models to perform the remapping of the bond lengths and bend angles by requiring that the average end-to-end distance is the same in both mappings. 
In the freely jointed chain model, the mean squared end-toend distance for a polymer consisting of $n$ monomers with bond length $l$ is given by

$$
\left\langle r^{2}\right\rangle=(n-1) l^{2}
$$

The freely rotating chain model specifies the bend angle $\theta$ between the bond vectors, which modifies the expression for the end-to-end distance as follows

$$
\left\langle r^{2}\right\rangle=(n-1) l^{2}\left[1+\frac{2 \alpha}{1-\alpha}-\frac{2 \alpha\left(1-\alpha^{n-1}\right)}{(n-1)(1-\alpha)^{2}}\right]
$$

where the exterior angle, $(\pi-\theta)$, is used to define $\alpha=$ $\cos (\pi-\theta)$.

By imposing the condition that the end-to-end distance is the same for both mappings, eq 3 gives

$$
\frac{l^{\prime}}{l}=\left(\frac{n-1}{\frac{n}{m}-1}\right)^{1 / 2}
$$

where the substitution $n^{\prime}=n / m$ has been made. The limit of infinite $n$ reduces to the simple expression

$$
\lim _{n \rightarrow \infty}\left(\frac{l^{\prime}}{l}\right)=\sqrt{m}
$$

Similarly, eq 4 gives

$$
\lim _{n \rightarrow \infty}\left(\alpha^{\prime}-\alpha\right)=0
$$

in the limit of infinite $n$, with $\alpha^{\prime}=\cos \left(\pi-\theta^{\prime}\right)$. A more detailed analysis establishes that the limit is approached as

$$
\alpha^{\prime}-\alpha \propto \frac{1}{n}
$$

Since the chain lengths considered in this work fall well into the large $n$ limit, the results given by eqs 6 and 7 were used to perform the remapping. As a result, only the bond parameters need to be changed.

This remapping is applied to the bonds in each of the polymer species, but at the diblock interface the bond connecting the two blocks also needs to be rescaled. However, for this interfacial bond the rescaling is minor because the effects of the two monomer mappings oppose each other. The rescaling is taken as

$$
\frac{l_{\mathrm{PEO}-\mathrm{PEE}}^{\prime}}{l_{\mathrm{PEO}-\mathrm{PEE}}}=\frac{\sqrt{m_{\mathrm{PEO}}}+\sqrt{m_{\mathrm{PEE}}}}{2}=1.03
$$

In addition to the rescaling of the mean values of the bonds the second moments must be rescaled to determine the force constants of the harmonic potentials that are used in DPD to describe the bonds. This was done according to the method used by Nielsen et al. ${ }^{39}$ in which the variance per unit length is kept constant, resulting in the force constant decreasing for longer bonds or increasing for shorter ones. The density mapping model parameters are presented in Figure 2 along with a schematic drawing of the mapping. Table 2 summarizes the target values and the values obtained with the optimized DPD parameters. Note that $l^{\prime}$ and $\theta^{\prime}$ correspond to the mean values presented in

\begin{tabular}{|c|c|c|c|c|}
\hline \multirow[b]{2}{*}{ bond } & \multicolumn{2}{|c|}{ AAMD } & \multicolumn{2}{|c|}{ DPD } \\
\hline & mean $(\AA)$ & $\operatorname{RMSD}(\AA)$ & $\overline{\text { mean }(\AA)}$ & $\operatorname{RMSD}(\AA)$ \\
\hline $\mathrm{H}-\mathrm{H}$ & 3.6021 & 0.1941 & 3.6339 & 0.2166 \\
\hline $\mathrm{H}-\mathrm{T}$ & 4.1812 & 0.2714 & 4.2609 & 0.2849 \\
\hline \multirow[t]{2}{*}{$\mathrm{T}-\mathrm{T}$} & 3.8770 & 0.5095 & 4.2138 & 0.4800 \\
\hline & \multicolumn{2}{|c|}{ AAMD } & \multicolumn{2}{|c|}{ DPD } \\
\hline bend & mean (rad) & RMSD (rad) & mean $(\mathrm{rad})$ & RMSD (rad) \\
\hline $\mathrm{H}-\mathrm{H}-\mathrm{H}$ & 2.0739 & 0.4427 & 2.0432 & 0.3672 \\
\hline $\mathrm{H}-\mathrm{H}-\mathrm{T}$ & 2.3945 & 0.4155 & 2.2806 & 0.3638 \\
\hline $\mathrm{H}-\mathrm{T}-\mathrm{T}$ & 1.3011 & 0.2678 & 1.4731 & 0.4056 \\
\hline $\mathrm{T}-\mathrm{T}-\mathrm{T}$ & 1.5306 & 0.3470 & 1.5869 & 0.4248 \\
\hline
\end{tabular}
the first column of Table 2 .

III.c. Intermolecular Interactions. The intermolecular parameters for the two mappings can each be determined
TABLE 2: Moments of the Probability Distributions for the Bonds and Bends Obtained from AAMD and DPD Simulations $^{a}$

${ }^{a}$ The intramolecular parameters shown in Figure 2 were chosen to achieve close agreement between these moments.

separately from experimental data. In the DPD framework ${ }^{31-35}$ the intermolecular pair interactions are represented as a sum of a dissipative force, a random force, and a conservative force. From these, only the latter depends on the type of atoms considered in each pair and is modeled as a soft repulsion that requires only one parameter, $a_{i j}$, for each pair to fully describe it. As seen in Figure 2, these parameters conform to a symmetric matrix from which the diagonal values are set to be $78 k_{\mathrm{B}} T$ to replicate the compressibility of water at room temperature. ${ }^{38} \mathrm{It}$ should be mentioned that instead of a remapping one could preserve the conventional mapping and attempt to change the $a_{i i}$ values such that agreement is obtained between the simulation and experimental bulk densities. This alternative solution was examined but gave a set of $a_{i i}$ values that were too big, which consequently started to influence the intramolecular parameters, hence, since the interaction terms were all coupled, it was not possible to converge to a satisfactory set of parameters.

The three off-diagonal terms are chosen such that the mutual solubilities are matched to the experimental values. As a consequence, these parameters are related to the interfacial tension between the two species considered, as shown by Maiti and McGrother. ${ }^{23}$ To obtain the $a_{\mathrm{TW}}$ repulsion parameter a DPD system consisting of a water/oil interface was simulated in order to replicate the experimental interfacial tension value of $\sim 50$ $\mathrm{mN} / \mathrm{m}$. Similarly, a simulation of a PEO/PEE interface was used to find the value of the repulsion parameter $a_{\mathrm{TH}}$ that would replicate the experimental PEO/PEE interfacial tension of $\sim 30$ $\mathrm{mN} / \mathrm{m}$.

A problem arises when the same method is applied to try to find the PEO-water repulsion parameter, $a_{\mathrm{HW}}$, because PEO is miscible in water at almost any concentration. For this case the observable to match was chosen to be the radial distribution function of $\mathrm{PEO}$ in water as measured from an AAMD simulation. The value obtained here, $a_{\mathrm{HW}}=79.3 k_{\mathrm{B}} T$, agrees with the value obtained by Groot and Rabone ${ }^{38}$ for the same two species by using the $\chi$-parameter extrapolated to room temperature from high-temperature demixing data.

\section{Structural and Mechanical Properties}

A representative snapshot from a DPD simulation of the hydrated PEO40-PEE37 bilayer is shown in Figure 3 along with the averaged density profiles for the two block copolymers and water normal to the bilayer plane. It is clear from the snapshot that there is a sharp interface between the hydrophobic core of the bilayer and the polar species in the system. The PEE density profile is localized near the bilayer midpoint and 


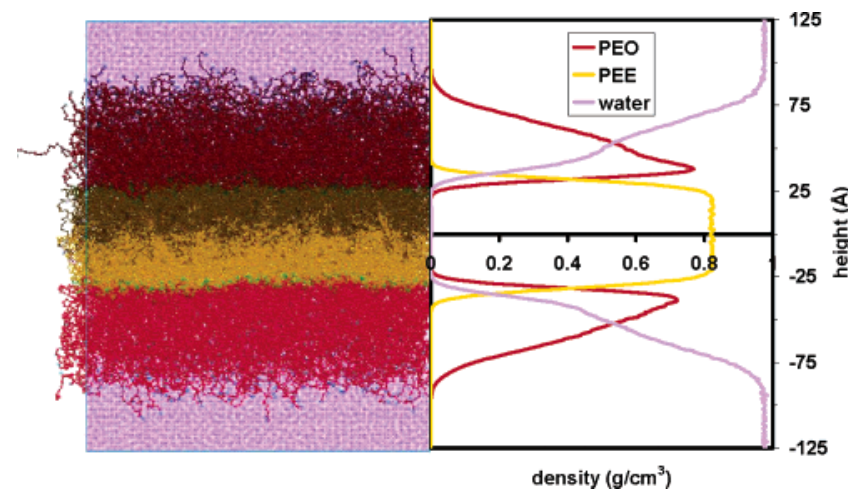

Figure 3. Bilayer snapshot and averaged bilayer species density profiles for PEO40-PEE37. In the snapshot, the difference in brightness between the two leaflets shows that, despite some entanglement, the two leaflets are largely distinct. The equilibrium density profiles are shown for PEO, PEE, and water.

it plateaus to a bulk density value of $0.82 \mathrm{~g} / \mathrm{cm}^{3}$, which is typical of hydrocarbons. This value is a direct result of using the density mapping described by Figure 2. The two leaflets are distinguished by brightness in the snapshot of Figure 3 to clearly show the extent of entanglement between the chains in the hydrophobic core. The PEO block is packed tightly against the hydrophobic core and extends well into the water subphase in which it is soluble. Indeed, the water density is observed to penetrate all the way through the hydrophilic block to the interface between the two blocks. Outside the bilayer the water density achieves its bulk value of $1 \mathrm{~g} / \mathrm{cm}^{3}$.

To verify that the model reproduces the mechanical properties of aqueous PEO-PEE systems, the area expansion modulus of the membrane, $K_{\mathrm{a}}$, was determined and compared to the value given in the literature. Figure 4A shows the results, where the equilibrium tension of the membrane is plotted as a function of the area expansion normalized by the area for which a zero tension is observed. The area expansion modulus can then be obtained directly from the slope of this curve giving a value of $137 \mathrm{mN} / \mathrm{m}$, which is in good agreement with the experimental value reported by Discher et al. ${ }^{5}$ of $120 \pm 20 \mathrm{mN} / \mathrm{m}$. Alternatively, if the data in Figure 4A are fitted to a line that crosses the origin the slope obtained is almost unchanged at $139 \mathrm{mN} / \mathrm{m}$.

\section{Scaling with Molecular Weight}

Since the model has proven to give correct values for the mechanical and structural properties of aqueous diblock copolymer systems, we are interested in seeing if the model is able to replicate the scaling behavior of hydrophobic core thickness with molecular weight of the hydrophobic block. As described in the Methods section the scaling of area per molecule, $A$, with molecular weight, $M$, of 0.5 suggested in the literature $^{16}$ was used to predict the values of the zero tension area for several diblock chain lengths. These predicted area values are given in Figure $4 \mathrm{~B}$ as the $x$-axis values for the triangles at each $M$, while the $y$-axis value gives the actual membrane tension obtained from a simulation performed at the predicted area. A second simulation performed for each $M$ at an area per molecule in the neighborhood of the predicted one gave the tension values represented by the diamonds in Figure 4B. A linear fit of the two data points obtained from these simulations was used (extrapolated if needed) to obtain the actual area per molecule. The straightforward way of doing the linear fit would be to calculate the slope and intercept of the line passing through the two points obtained from the simulations.

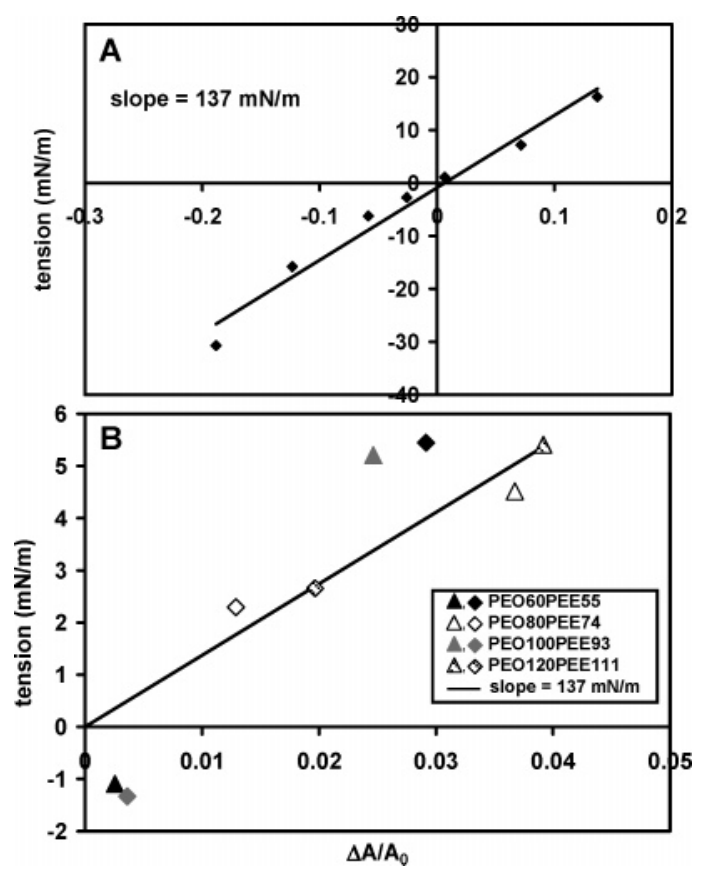

Figure 4. (A) Tension versus area expansion for the PEO40-PEE37 bilayer. The tensionless state, $A_{0}$, is determined from a plot of tension versus area. Transforming from the area $A$ to the normalized difference $\left(A-A_{0}\right) / A_{0}$ yields the plot shown here, the slope of which gives the area expansion modulus $K_{\mathrm{a}}$ of $137 \mathrm{mN} / \mathrm{m}$. The experimental value is reported as $K_{\mathrm{a}}=120 \pm 20 \mathrm{mN} / \mathrm{m}$ (Discher et al. Science 1999, 284, 1143). (B) The scaling law $A \sim M^{1 / 2}$, where $A$ is the tensionless area and $M$ is the hydrophobic molecular weight, is used together with the PEO40-PEE37 data to make an initial prediction for the tensionless area, shown in triangles, for the different molecular weight diblock copolymers shown in Table 1. Simulations at those predicted areas gave tensions corresponding to $x$-axis values given by the triangles. Based on these values, one additional simulation for each size, shown in diamonds, is performed to refine the initial guess by linear interpolation with the restriction of constant $K_{\mathrm{a}}$ as given by part A.

Although this method gives a reasonable scaling of area per chain with molecular weight of 0.46 , we consider it overweights the two data points in the following sense: it has been shown ${ }^{13,16}$ that $K_{\mathrm{a}}$ of the membrane is independent of the molecular weight of the chains. A more suitable fit would be done by assuming a constant slope of $137 \mathrm{mN} / \mathrm{m}$, which was the value obtained from seven data points for the PEO40-PEE37 system. The constant $K_{\mathrm{a}}$ fit, shown in Figure 4B, gives roughly the same scaling law with molecular weight of 0.46 (not shown), consistent with the experimental value of 0.5 .

In addition, from the simulations of different chain lengths, the hydrophobic core thickness was obtained as the distance between the two $z$-values at which the PEE density was $10 \%$ of its bulk density of $0.82 \mathrm{~g} / \mathrm{cm}^{3}$ ( $z$ is taken as the dimension normal to the bilayer). The thickness was then calculated for each chain length by substituting the zero tension area into the linear fit obtained for a plot of thickness versus area per molecule. These values are plotted versus molecular weight of the hydrophobic block in Figure 5. A power fit to these data gives a scaling of 0.48 , a value that agrees within experimental uncertainty with the reported value of 0.5 , which is also shown in Figure 5 as a dotted line.

\section{Vesicle Rupture}

As an illustration of the system sizes routinely accessible with DPD, we present preliminary studies of vesicle rupture. Polymersome poration is of great interest as a mechanism for the 


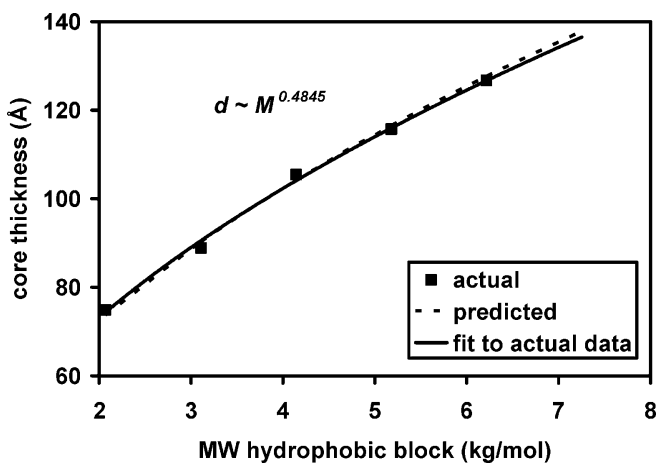

Figure 5. Scaling of hydrophobic core thickness $d$ with hydrophobic molecular weight $M$. The experimentally observed scaling law of $d \sim$ $M^{1 / 2}$ is shown along with the DPD data, which scales such as $d \sim$ $M^{0.48}$, a value within the experimental uncertainty.
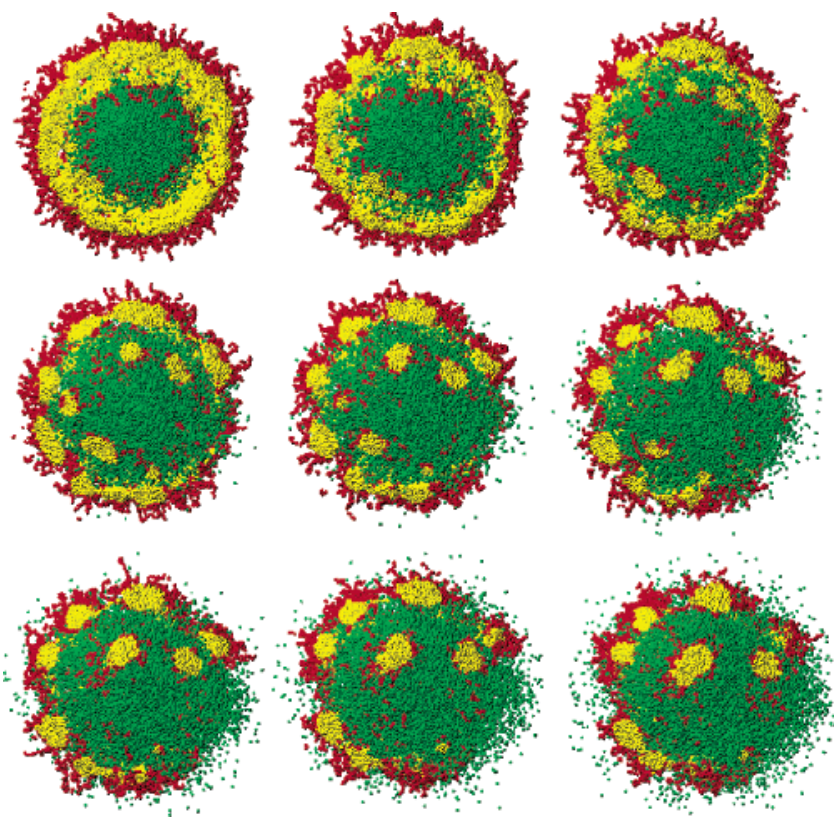

Figure 6. Sequence of cross-sections equally spaced in time of a vesicle rupture event simulated with DPD. The inner leaflet micellizes first, triggering the rapid micellization of the outer leaflet. The snapshots show the hydrophobic PEE blocks in yellow, the hydrophilic PEO blocks in red, and the internal water in green (exterior water is not shown).

controlled release of molecules sequestered in the vesicle interior. This release is achieved either by applying an electric field $^{40}$ (electroporation) that transiently porates the vesicle or by incorporating degradable polymer into the vesicle ${ }^{41}$ which leads to irreversible poration and rupture with a characteristic time scale set by the amount of degradable polymer used. Although not the focus of this work, it is felt that the DPD technique is suitable for the study of vesicle rupture and a detailed analysis of poration pathways and comparison to experiments will be considered for future study.

The evolution of a vesicle with an expanded area per diblock is shown in Figure 6 with a time interval of 2000 time steps between the snapshots. This setup can be directly related to osmotic swelling experiments in which an osmotic pressure gradient is induced by changing the solvent concentration across the vesicle membrane. The simulation suggests that a possible poration mechanism involves disruption and micellization of the inner leaflet, exposing the hydrophobic core to the solvent. The outer leaflet responds to this exposure by collapsing into numerous micelles, allowing the vesicle contents to escape (shown in green in Figure 6). The size chosen for this system is smaller than typically found in experiments, resulting in a vesicle with high curvature that could have an effect on the poration pathway. Proper characterization of polymersomes can be achieved by simulating lower curvature vesicles which are still accessible by DPD.

\section{Discussion and Conclusions}

The DPD model proves to be effective at capturing the experimental scaling relations while maintaining simplicity in the molecular description of aqueous diblock copolymer membranes by only incorporating the effects of the tendency of the components to phase separate and the chemical bond attaching the blocks together. However, a modification of the standard DPD coarse graining was required to obtain a physically reasonable description of these systems. The mapping criterion was modified from a uniform density set by the water to a variable species-dependent density. The resulting bond lengths between adjacent polymer beads were determined from the standard mapping by appealing to the freely jointed chain model. These bond lengths were chosen to be independent of the chain length (molecular weight) of the polymer being modeled and hence do not influence the scaling exponents reported in this work.

Historically, the intramolecular models developed in the DPD scheme were parametrized in an arbitrary manner. In more recent works a stronger connection has been made to atomistic simulations by using the appropriately coarse-grained bond length and bend angle distributions. ${ }^{42,43} \mathrm{We}$ continued in this vein by using atomistic simulations to build the intramolecular target distributions. Similar target distributions assembled from the same underlying atomistic molecular dynamics simulations were used to construct a coarse-grain molecular dynamics model $^{12,13}$ that has also been shown to be in good agreement with experimental data. These constructions both reinforce the integrity of each other and lead to the ability to study diblock copolymer vesicles over multiple length and time scales (see Figure 1).

Future extensions are anticipated to proceed in two complementary directions. On the simulation side, the two other dominant aqueous morphologies of wormlike (cylindrical) and spherical micelles should be simulated and compared to the existing experimental data to verify the model and supplement the experimental scaling relations which have not been unambiguously determined. In addition, the time and length scales achievable with DPD should be used to characterize polymer vesicles of experimentally relevant sizes. On the theoretical side, the hydrophobic domain size scaling with molecular weight for bilayers, worm micelles, and spherical micelles should be derived. Semenov's strong segregation limit theory for melts predicts a scaling exponent of $2 / 3$ for all three morphologies, which results from a balance between the domain free energy and the interfacial energy contributions. ${ }^{15}$ Extensions of Semenov's theory to monolayers of diblock copolymers adsorbed at the interface between liquids formed from the two separate blocks report different scaling exponents. Wang and Safran ${ }^{44}$ found that, in the long chain limit, the scaling can range from $2 / 5$ to $2 / 3$ depending on the excluded volume interactions and the correlations between the chains. The two treatments of chain correlation considered by Wang and Safran, namely mean field and semidilute, yield similar exponents. However, different considerations of the excluded volume effect yield large changes in the scaling. For a melt system, defined as a small size difference between the diblock and the solvent, the scaling reported was $2 / 3$, while for a swollen system, defined as a large 
size difference between the diblock and the solvent, the scaling was found to be $2 / 5$. The mixed monolayer case, in which one block is swollen and the other block is in a melt, is appropriate for describing hydrated bilayer membranes because for bilayers the hydrophobic block is sequestered from the solvent, while the hydrophilic block is swollen with water. It is expected that this case will show an exponent between the two pure cases depending on the relative influence of the two sides of the interface, but this is not trivial to solve because the influence of each case on the curvature elastic free-energy expression is sensitive to the morphology being considered. This is consistent with experimental results that show different scalings for different morphologies, ${ }^{16,45}$ but the small curvature expansion used in the theoretical treatment is not very meaningful for the high curvature morphologies observed in experiments. This theory is a step in the right direction and we hope that current and future experimental and simulation data will encourage further theoretical developments to achieve a more complete microscopic physical understanding of aqueous diblock copolymer systems.

Acknowledgment. Stimulating discussions with Berend Smit on the density mappings are gratefully acknowledged. Funding for this work has being provided by MPIKG and NSF funded IGERT (Grant DGE-0221664) and MRSEC programs.

\section{References and Notes}

(1) Bangham, A. D.; Haydon, D. A. Br. Med. Bull. 1968, 24, 124.

(2) Johnson, S. M.; Bangham, A. D. Biochim. Biophys. Acta 1969, $193,92$.

(3) Egberts, E.; Berendsen, H. J. C. J. Chem. Phys. 1988, 89, 3718.

(4) Gronowski, A. A.; Jiang, M.; Yeager, H. L.; Wu, G.; Eisenberg, A. J. Membr. Sci. 1993, 82, 83 .

(5) Discher, B. M.; Won, Y. Y.; Ege, D. S.; Lee, J. C. M.; Bates, F.

S.; Discher, D. E.; Hammer, D. A. Science 1999, 284, 1143.

(6) Cornelissen, J.; Fischer, M.; Sommerdijk, N.; Nolte, R. J. M. Science 1998, $280,1427$.

(7) Vanhest, J. C. M.; Delnoye, D. A. P.; Baars, M.; Vangenderen, M. H. P.; Meijer, E. W. Science 1995, 268, 1592.

(8) Kramer, E.; Forster, S.; Goltner, C.; Antonietti, M. Langmuir 1998 14, 2027.

(9) Nardin, C.; Hirt, T.; Leukel, J.; Meier, W. Langmuir 2000, 16, 1035.

(10) Kaler, E. W.; Murthy, A. K.; Rodriguez, B. E.; Zasadzinski, J. A N. Science 1989, 245, 1371.

(11) Muller, M.; Schick, M. J. Chem. Phys. 1996, 105, 8282.
(12) Srinivas, G.; Shelley, J. C.; Nielsen, S. O.; Discher, D. E.; Klein, M. L. J. Phys. Chem. B 2004, 108, 8153.

(13) Srinivas, G.; Discher, D. E.; Klein, M. L. Nat. Mater. 2004, 3 , 638.

(14) Ahmed, F.; Hategan, A.; Discher, D. E.; Discher, B. M. Langmuir 2003, 19, 6505

(15) Bates, F. S.; Fredrickson, G. H. Annu. Rev. Phys. Chem. 1990, 41, 525 .

(16) Bermudez, H.; Brannan, A. K.; Hammer, D. A.; Bates, F. S.; Discher, D. E. Macromolecules 2002, 35, 8203.

(17) Jain, S.; Bates, F. S. Science 2003, 300, 460.

(18) Pei, Z. C.; Pei, Y. X.; Wang, Q. R. J. Appl. Polym. Sci. 2002, 85 2521 .

(19) Dalhaimer, P.; Bates, F. S.; Aranda-Espinoza, H.; Discher, D. C. R. Phys. 2003, 4, 251.

(20) Photos, P. J.; Bacakova, L.; Discher, B.; Bates, F. S.; Discher, D. E. J. Controlled Release 2003, 90, 323.

(21) Opsteen, J. A.; Cornelissen, J.; van Hest, J. C. M. Pure Appl. Chem. 2004, 76, 1309.

(22) Frenkel, D.; Smit, B. Understanding Molecular Simulations: From Algorithms to Applications, 2nd ed.; Academic Press: San Diego, CA, 2002.

(23) Maiti, A.; McGrother, S. J. Chem. Phys. 2004, 120, 1594.

(24) Nielsen, S. O.; Lopez, C. F.; Srinivas, G.; Klein, M. L. J. Phys. Condens. Matter 2004, 16, R481.

(25) Shillcock, J. C.; Lipowsky, R. J. Chem. Phys. 2002, 117, 5048.

(26) Kranenburg, M.; Venturoli, M.; Smit, B. J. Phys. Chem. B 2003 $107,11491$.

(27) Shillcock, J. C.; Lipowsky, R. Nat. Mater. 2005, 4, 225.

(28) Groot, R. D.; Warren, P. B. J. Chem. Phys. 1997, 107, 4423.

(29) Groot, R. D.; Madden, T. J. J. Chem. Phys. 1998, 108, 8713

(30) Jones, J. L.; Lal, M.; Ruddock, J. N.; Spenley, N. A. Faraday Discuss. 1999, 129.

(31) Hoogerbrugge, P. J.; Koelman, J. Europhys. Lett. 1992, 19, 155.

(32) Espanol, P.; Warren, P. Europhys. Lett. 1995, 30, 191.

(33) Marsh, C. A.; Backx, G.; Ernst, M. H. Phys. Rev. E 1997, 56, 1676.

(34) Espanol, P. Europhys. Lett. 1997, 40, 631.

(35) Pagonabarraga, I.; Hagen, M. H. J.; Frenkel, D. Europhys. Lett 1998, $42,377$.

(36) Jakobsen, A. F.; Mouritsen, O. G.; Besold, G. J. Chem. Phys. 2005 $122,204901$.

(37) Goetz, R.; Lipowsky, R. J. Chem. Phys. 1998, 108, 7397.

(38) Groot, R. D.; Rabone, K. L. Biophys. J. 2001, 81, 725.

(39) Nielsen, S. O.; Lopez, C. F.; Srinivas, G.; Klein, M. L. J. Chem Phys. 2003, 119, 7043.

(40) Neumann, E. Electroporation and Electrofusion in Cell Biology; Plenum: New York, 1989.

(41) Ahmed, F.; Discher, D. E. J. Controlled Release 2004, 96, 37.

(42) Kranenburg, M.; Nicolas, J. P.; Smit, B. Phys. Chem. Chem. Phys. 2004, 6, 4142 .

(43) Li, D. W.; Liu, X. Y.; Feng, Y. P. J. Phys. Chem. B 2004, 108, 11206

(44) Wang, Z. G.; Safran, S. A. J. Chem. Phys. 1991, 94, 679.

(45) Dalhaimer, P.; Bermudez, H.; Discher, D. E. J. Polym. Sci., Part B: Polym. Phys. 2004, 42, 168. 